\section{ADVANCED HUMAN-COMPUTER INTERFACES FOR AIR TRAFFIC MANAGEMENT AND SIMULATION}

\author{
Ronald Azuma and Mike Daily \\ Hughes Research Laboratories \\ 3011 Malibu Canyon Blvd. MS RL96 \\ Malibu, CA 90265 \\ Jimmy Krozel \\ Seagull Technology \\ 21771 Stevens Creek Blvd. \\ Cupertino, CA 95014-1175
}

\begin{abstract}
New technologies will significantly change Air Traffic Control over the next 15 years. These changes will require improved human-computer interfaces for the less regulated, more complex future environment. This paper describes a highly interactive, real time demonstration of 3-D visualization and interface concepts for the air traffic domain, including Free Flight. This demonstration offers a 3-D, stereoscopic view from both the controller's and pilot's perspectives, featuring representations of projected flight paths, 3-D graphical and audio proximity warnings, and 3-D text labels that automatically reorient themselves. Feedback from domain experts is described. In Free Flight, pilots and airlines will set their own courses and resolve conflicts autonomously when possible. This demonstration also shows visualizations of the Protected Airspace Zone and Tactical Alert Zone safety regions around Free Flight aircraft, which are most easily understood through the use of 3-D graphics. Future versions of this demonstration will acquire more realistic data, improve the interaction techniques and integrate the visualization more closely with conflict detection and resolution algorithms.
\end{abstract}

\section{Motivation}

During the next 15 years, Air Traffic Control (ATC) systems will undergo significant changes due to new technologies ${ }^{16}$. Such changes are required to meet the expected growth in air traffic. New technologies such as the Global Positioning System (GPS), Automatic

Copyright (C) 1996 by the American Institute of Aeronautics and Astronautics, Inc. All rights reserved.
Dependent Surveillance (ADS) communications, and more sophisticated ATC software will provide some of these required improvements. These technologies will establish an "Internet in the sky," providing an information-rich environment for distributing many types of data amongst pilots and controllers. However, these technologies by themselves will not satisfy all the needs of future ATC systems. Another vital but sometimes overlooked component is the humancomputer interface: how controllers and pilots will interact with the information provided by these new technologies. Despite increased automation and more sophisticated computers and sensors, humans will always be "in the loop." People, not computers, fly the aircraft, direct the traffic, and have the final word in all decisions. Therefore, how computer systems present information to the human controllers and pilots will play a crucial role in the effectiveness of future ATC systems.

While existing human-computer interfaces may be sufficient for today's ATC environment, they will not be in the future when air traffic patterns will be more complicated and less ordered. Existing controller interfaces use flat, 2-D displays with trackball and button-based controls. Pilots may have no traffic displays at all, relying on charts and voice commands from controllers. However, the advent of Free Flight will change the current situation dramatically. Under certain conditions, Free Flight allows pilots to set their own course and resolve conflicts by themselves, without involving controllers except when needed. This distributes the task of air traffic control over all aircraft, reducing the air traffic controllers' workloads. However, instead of the orderly well-regulated traffic patterns that exist today, there will be the potential for more complex and variable traffic patterns. Predicting and avoiding conflicts in a such an environment will be more difficult than it is today. More importantly, airlines will take an active role in air traffic management. Therefore, pilots and Airline Operation Centers (AOCs) must also have displays that show the ATC situation and potential conflicts and solutions in a way that is easy to quickly understand, without drawing concentration away from the task of flying the aircraft. Existing interfaces will not meet these needs for either pilots or controllers.

We believe the best course for meeting these future needs is to combine automated conflict detection and resolution mechanisms with advanced human-computer interfaces that use intuitive and natural threedimensional displays and controls, such as those being developed in Virtual Reality systems. Such displays 
offer the potential to reduce the cognitive workload on both pilots and controllers, resulting in faster recognition and improved situational awareness of the air traffic situation and potential conflicts. These displays will be multimodal, engaging the visual, auditory and vocal channels to provide an interface that fits more naturally to the way humans normally operate, rather than forcing the human to meet the demands of the computer. The air traffic management domain provides a rich environment to study the potential of these multimodal interface techniques. This domain requires the simulation and display of a multiple aircraft airspace, intent data, proximity information for aircraft conflict detection, and conflict resolution options. In this paper, we present multimodal display techniques aiding situation understanding of the pilot and air traffic controller.

\section{Contribution}

Several investigators have applied 3-D visualization and virtual reality techniques to the air traffic management problem. Several recent studies $5,8,9,11,12$ have investigated the potential benefit of 3-D or perspective displays to aid specific ATC tasks. One study $^{17}$ showed that when a display does not show the third spatial dimension as readily apparent as the other two, pilots tend to solve conflict avoidance problems in the displayed two directions more often than in the three dimensions. For conflict resolution, this implies that a plan view display may bias conflict resolution solutions to right and left turning maneuvers. A followon study ${ }^{8}$ provided a perspective display to pilots for traffic avoidance and found the pilots were more likely to choose a solution with a vertical component. At least two groups have used virtual reality interfaces to examine ATC issues ${ }^{3,4,20}$. Pruyn ${ }^{15}$ explored and demonstrated several concepts for applying 3-D visualization techniques to the ATC environment.

The contribution of this paper lies in a highly interactive, real-time demonstration of some concepts that we have not seen previously demonstrated in the ATC environment and in the lessons learned about these ideas. This is especially true of our concepts as applied to the Free Flight domain, which we have not seen discussed in any previous visualization effort. We introduced some of our concepts in a previous paper ${ }^{7}$; the difference here is the actual implementation, demonstration and evaluation of those concepts. Our demonstration code is based on an original version from the MIT Media Lab ${ }^{18}$, which was greatly modified and extended by the authors to demonstrate our ideas.

Our approach differs from some previous works in that our goal is to create and demonstrate visualization concepts for the ATC domain, receive rapid feedback from domain experts, and then repeat the cycle. We have not been running user studies on incremental improvements from traditional 2-D ATC displays. We believe rapid prototyping followed by rapid evaluation is a better approach to find the more advanced visualization concepts that seem to be beneficial, which can then be formally evaluated by user studies. Furthermore, we have restricted our use of specialized equipment to the minimum required for demonstrating our concepts. While other virtual reality efforts use Head-Mounted Displays (HMDs) and fully-immersive displays, we have avoided that in favor of a stereo display on a low-end graphics workstation, even though our laboratory is equipped to run HMDs on expensive graphics engines. In this way, we have reduced the "culture shock" of exposing our ideas to controllers and pilots who are used to flat, 2-D display panels and increased the likelihood of eventual implementation of some of our concepts, due to lower cost requirements.

\section{Initial Demonstration}

The initial demonstration system is shown in Figure 1. The graphics engine is a low-cost Silicon Graphics (SGI) Indy. The user views the monitor and sees stereo images through a pair of Crystal Eyes stereo glasses, made by Stereographics. The user controls the demo through a mouse-based interface. 3-D sound is generated by two Alphatron sound boards, made by Crystal River Engineering. These boards run in a '486 PC, connected to the Indy through a serial line. The synthesized 3-D sound is routed to an audio amplifier and broadcast to a pair of $900 \mathrm{MHz}$ wireless radio headphones.
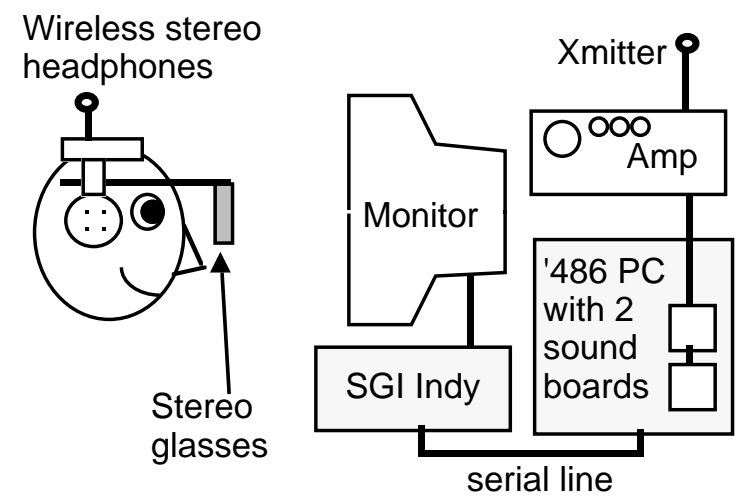

Figure 1: System diagram. 


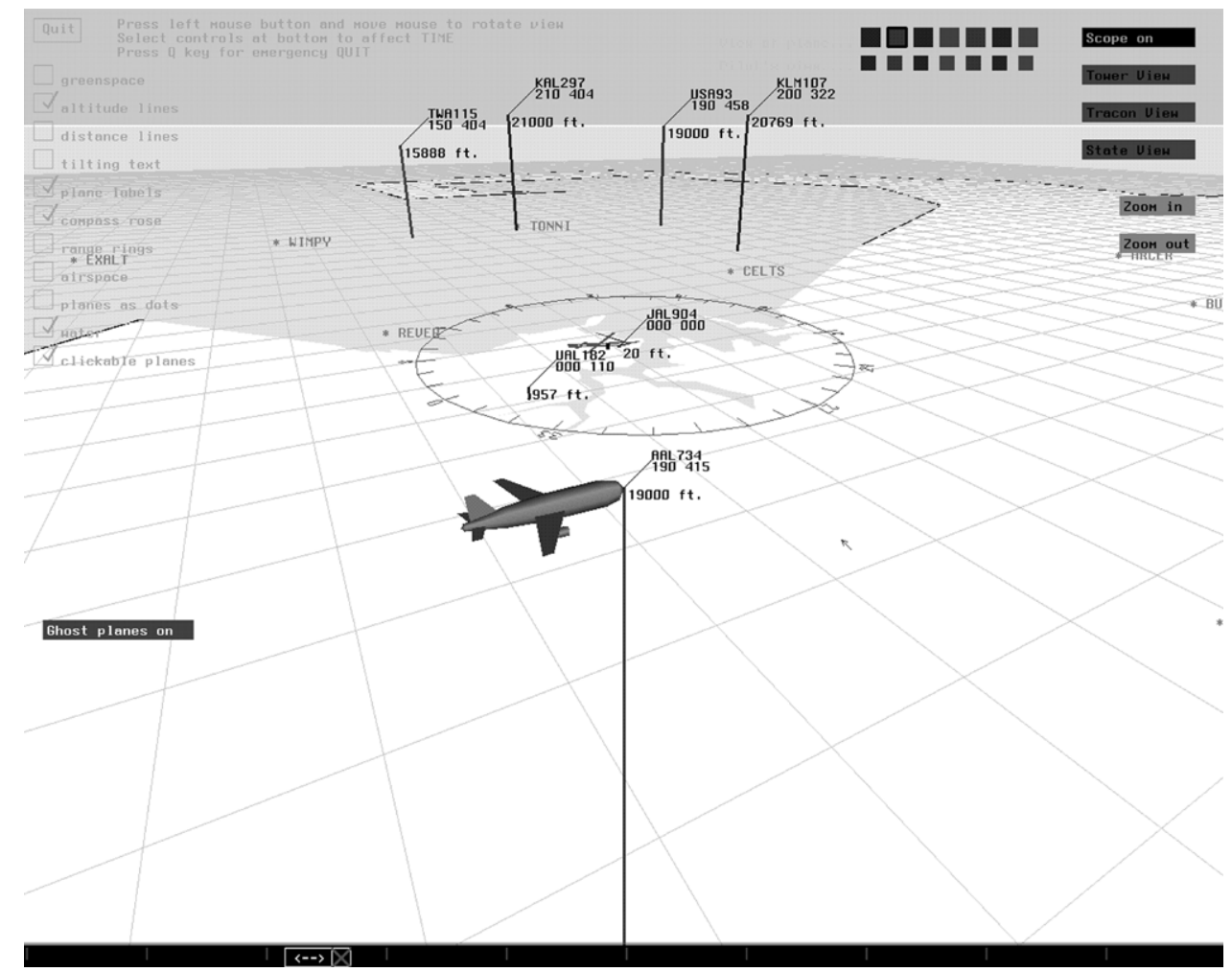

Figure 2: A sample view of what the user sees.

We first demonstrated this system on the exhibit floor of the 1995 Air Traffic Control Association Conference ${ }^{1}$. Figure 2 shows a typical scene from the demonstration. This system demonstrated the following features:

- 3-D Perspective Display: Standard ATC displays show the situation in a top-down, plan-view 2-D format. This system provides that mode but also allows the user to navigate and view the scene in 3-D. The view can be exocentric, looking at the entire situation from a remote perspective, or egocentric, following an individual aircraft to see the pilot's perspective. The user controls the point of view with a mouse, using a "virtual trackball" metaphor to change the pitch and yaw of the gaze direction. Simply clicking on an aircraft or other object in the environment causes the system to focus the user's view on that object. Objects that can be clicked are indicated by pulsating 2-D icons that appear over the 3-D object when the cursor is moved over that object. Zooming the viewpoint towards or away from an object is done by pushing the appropriate buttons. The visuals can be displayed in stereo, further enhancing the 3-D effect. To provide additional depth cues, altitude lines and shadows indicate the position of each aircraft projected onto the ground. The entire database represents an area around Boston's Logan airport. The display runs at interactive rates, varying from $5-20 \mathrm{~Hz}$ depending on which visualization features are activated.

Certain rendering techniques are used to achieve interactive rates on a low-cost platform. Hiddensurface removal is performed primarily by using a painter's algorithm. Z-buffering is only applied to the small areas covered by the 3-D aircraft models. Textures are not supported in hardware, so grid lines are used instead to provide context for aircraft motion relative to the ground. The total polygon and line counts are kept low, and simplified models are used to render distant objects in a "level of detail" scheme.

- Spatial and Temporal Continuity: When the user changes viewpoints, transitions are handled smoothly. Instead of immediately changing from the current viewpoint to the selected new viewpoint, the system automatically interpolates between the old and new viewpoints. This transition takes 1-2 seconds and uses the "slow in, slow out" metaphor that slows down the rate of transition as the user leaves the initial viewpoint and arrives at the new viewpoint. Smooth viewpoint transitions aid spatial awareness by helping the user retain context with respect to the overall situation.

- Object-Oriented Text: In existing ATC displays, 2-D text labels are attached to aircraft and other objects. 
This program also supports that mode by superimposing 2-D labels on top of their associated 3-D objects. However, another way of displaying the same information is to draw the text as 3-D objects near the labelled object. Users may perceive 3-D text labels to be more closely associated with their objects than with 2-D text labels. To be readable, these 3-D labels automatically reorient and reposition themselves based upon the user's viewpoint with respect to the object. That way, the user never has to read text that is backwards or upside down. This is done by computing the orientation of the object with respect to the current viewpoint and adjusting the label orientation and position appropriately. Figures 3 and 4 show a 3-D label (the aircraft's call sign) attached to an aircraft as viewed from two different viewpoints, demonstrating how the text automatically reorients itself to remain legible. Figure 5 shows 3-D runway labels on the model of Boston Logan.

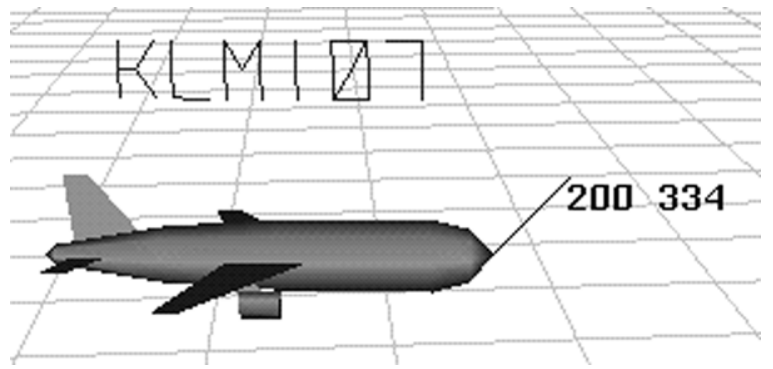

Figure 3: 3-D text label attached to aircraft.

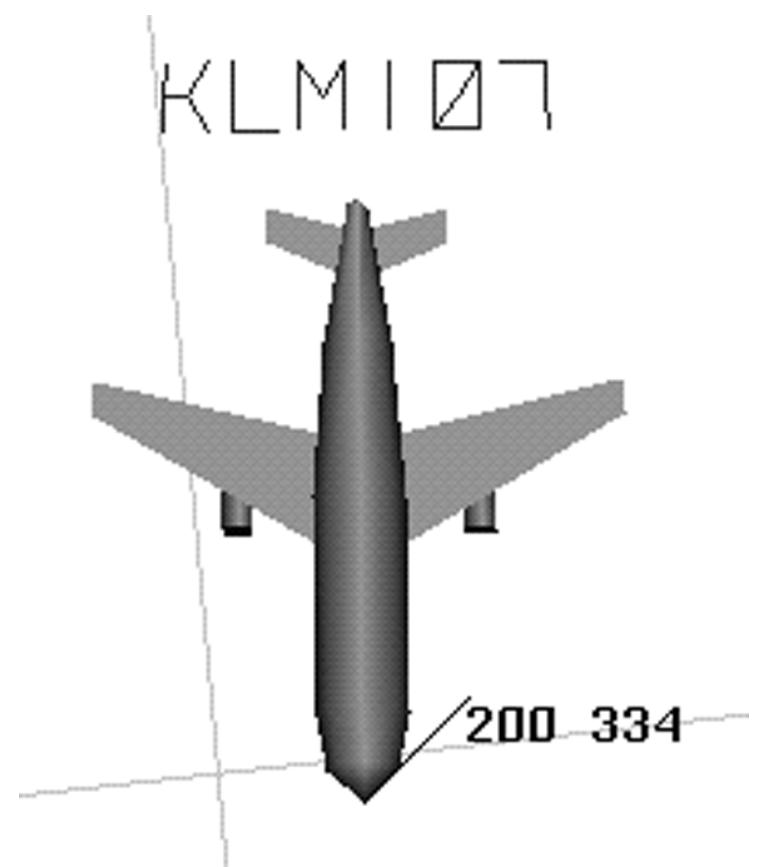

Figure 4: Reoriented 3-D text at a new viewpoint.

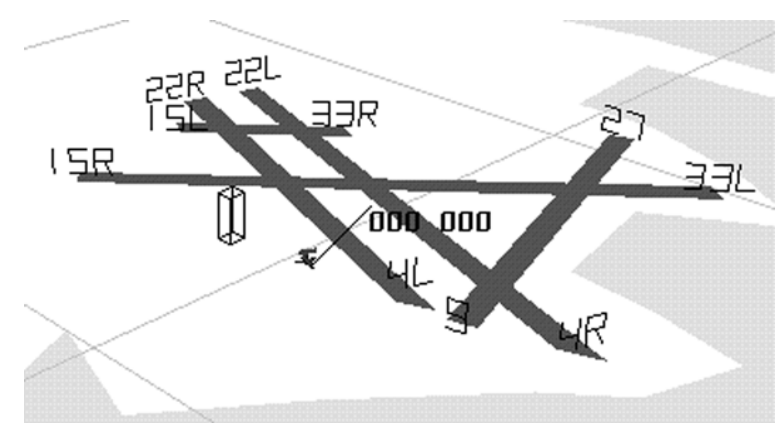

Figure 5: 3-D runway labels on Boston Logan.

One potential drawback to written information in 3-D is reduced precision in reading values along any one particular axis ${ }^{19}$. With object-oriented text, the orientation of the text is modified based on the viewing angle, reducing the effect of this potential problem. However, another issue that must be addressed is whether the text should remain at a fixed size or vary in size with respect to the distance from the viewpoint to the text. In this demo, the text remains at a constant size, but it could be changed to remain roughly constant in apparent screen area by scaling the 3-D font size. This prevents nearby text from occluding nearby objects and distant text from becoming illegible. Scaling the font size may also have a reinforcing effect with other motion cues.

- Visualization of Flight Paths: In the future, aircraft equipped with GPS antennas and ADS communications will be able to broadcast their position and intended path to control towers and other aircraft. This leads to the possibility of visualizing this information to help foresee future conflicts. This demonstration displays past and future flight paths through the use of a "ghost plane" metaphor. Ghost planes are projected future or past versions of aircraft, drawn as wireframe objects to avoid confusion with the actual aircraft (see Figure 6). Users can adjust how far into the future or past the ghost planes are projected. Since the flight paths for all aircraft are predetermined in this demo, both past and future projections use the actual paths. In reality, future projections would combine extrapolated trajectories with intent data. A wireframe line between the ghost plane and actual aircraft helps associate the two and the projected course of the aircraft (Figure 7). The ghost plane information is drawn in four different modes: static, shooting, pulsing, and airspace. Static mode simply puts the ghost plane at the projected location. Shooting mode repeatedly "shoots" the ghost plane from the actual aircraft to the projected position, following the projected path. Pulsing mode is similar, except that the ghost planes move forward and backward in time in a sinusoidal pulsing motion. By 
adding temporal motion, these two modes help users see how groups of aircraft will move in space and time. Finally, airspace mode represents a "highway in the sky" by drawing a set of linked wireframe rings in space that extend out as far as the projected ghost plane position (Figure 8). The result is a tunnel in the sky that the aircraft appears to fly towards (or away from), with the tunnel constantly staying ahead (or behind) the aircraft. This is useful in conflict detection by showing whether or not the airspace of two aircraft will intersect in the future, indicating a potential conflict.

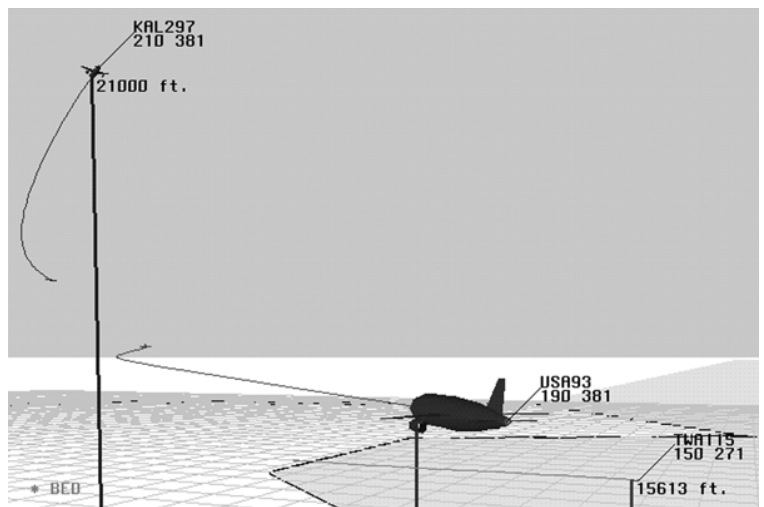

Figure 7: Several ghost plane future paths.

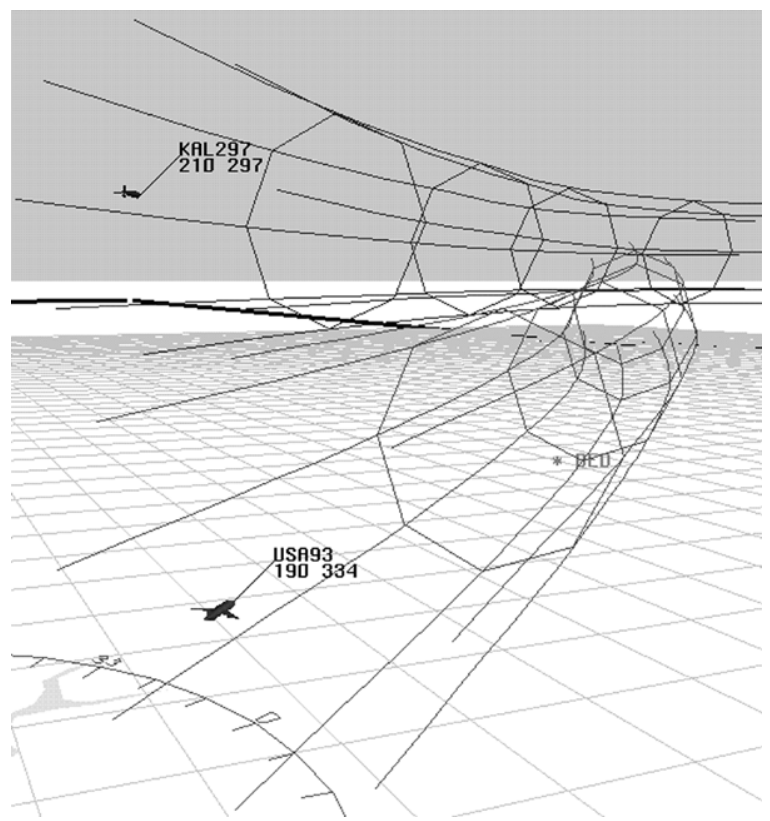

Figure 8: Ghost plane airspace mode.

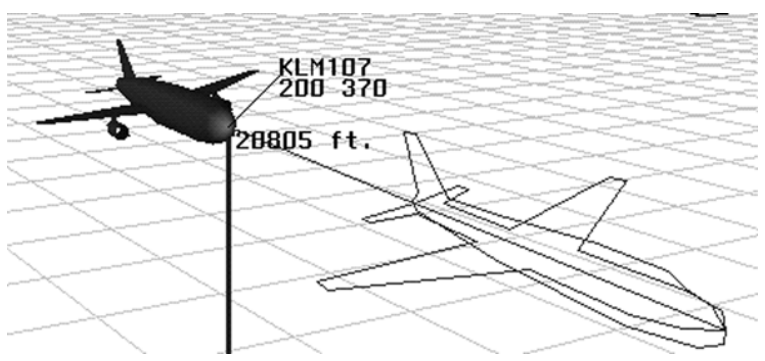

Figure 6: Ghost plane in front of actual aircraft.

- 3-D Spatial Audio Cues: The use of 3-D sound generation hardware allows the system to attach sounds to specific objects in space. This is more than just stereo. The sounds appear to emanate from their 3-D locations, giving an additional cue for the locations of certain objects. By offering multimodal displays, users can keep track of the rough location of an object even when it leaves the visual field of view. Since we do not perform head tracking in this system, the 3-D sound computations assume the user looks straight at the workstation screen at all times. 3-D sound is used in three separate modes in this demonstration.

First, it is used as an audio alert to identify nearby aircraft. The program has a mode called "greenspace" that draws color-coded lines from the current aircraft being gazed at to other nearby aircraft: green for far away, yellow for medium distance, and red for too close. 3-D audio alerts supplement these graphic warnings, where the audio alerts are attached in space to the other nearby aircraft. 3-D audio alerts are a natural way of presenting warnings. An automobile driver is usually first aware of the presence of an ambulance by the wail of its siren and roughly estimates the location of the ambulance using sound cues. Similarly, in experiments with TCAS II collision avoidance logic, 3-D spatialized sound simulated with pilot headphones allowed pilots to acquire conflict aircraft targets approximately 2.2 seconds faster than crew members who used monotone one-earpiece headsets ${ }^{2}$.

The second 3-D sound mode attaches an audio source to the end of one of the runways at Boston Logan. Pilots can use this as an audio beacon for keeping track of the location of a critical object in space (e.g. an airport location, a military no-fly zone, etc.) without having to look at it.

The third and last 3-D sound mode demonstrates the ability of 3-D sound to help users sort out confusing audio situations. While the user looks out at the scene from inside the control tower at Boston Logan, four 
conversations are played simultaneously. First, the conversations are played in mono so they all overlap each other. Listeners find it extremely difficult to focus on any of the conversations. Then we spatialize the sounds, placing each of the four conversations on one of the four main windows in the control tower (so they are separated by 90 degrees from their neighbors). As the user rotates his view within the tower, the conversations appear to rotate around his head. This takes advantage of the "cocktail party" effect: the ability to focus your hearing on a conversation that comes from a particular direction in space. Listeners find it much easier to select one conversation and focus on that with spatialized sound.

\section{Feedback and Lessons Learned}

We have not run user studies to measure the effectiveness of our ideas. Instead, we sought feedback from domain experts (controllers and pilots) who experienced the demo ${ }^{1}$. We learned several lessons:

- Pilots and controllers provided different feedback: Most controllers liked the ability to choose a static 3-D perspective view to watch the traffic from, but they did not seem to care about the interactive navigation techniques or most of the other features in the demo. A few were adamant that the 3-D displays were bad ideas and too confusing. One controller did suggest the use of 3-D sound for directing ground traffic. By making a pilot's voice appear to come from the direction of the aircraft's position on the runway, a controller issuing commands would gain an additional spatial cue to help him keep track of where the aircraft were on the runways, reducing the chance of issuing the wrong set of orders. Controllers suggested using these features in training new controllers, to help them build a "mental model" of the 3-D nature of the airspace. A few suggested it might speed up transition time when changing controllers at a station, where the incoming controller has to spend some time watching displays to understand the 3-D situation before he can relieve the controller on duty.

Pilots, on the other hand, liked most of the features, especially the visualization of future flight paths using "ghost planes" and the spatial cues for indicating neighboring aircraft. Reaction to the 3-D sound was mixed: some thought it would be useful, but others found it irritating. Military personnel thought 3-D displays could be useful for communicating information about enemy aircraft, targets on the ground, etc. that came from other sensors, such as an AWACS aircraft. Pilots suggested using this type of display in "Free
Flight," when pilots will have more authority to choose their own flight path and resolve potential conflicts without the direct intervention of controllers.

These different reactions are probably due to the different requirements and biases in the two professions. Controllers train on and use 2-D displays with fixed viewpoints (no zoom capabilities) that help them quickly and consistently estimate critical distances, looking at the environment from an exocentric or "god's eye" view. This may cause a bias against 3-D displays with changing viewpoints. Pilots are used to egocentric viewpoints that see the environment from the point of view of a specific aircraft, so they were more accepting of those display modes and the attempts to aid situational awareness from the pilot's perspective.

- Weather and terrain features: This demo does not model weather or any terrain features. Both are factors significantly affecting air traffic and need to be considered for future visualization efforts.

- Motion is vital for 3-D sound: An automobile driver who hears an approaching ambulance will rotate his head to locate the siren's origin. Without motion, people have a more difficult time locating the source of a sound. Having controls that let the user rapidly change the orientation of his viewpoint was important for making 3-D sound work. Ideally, head tracking should also cue sound changes as the user turns his head.

- Overload: Displays and interfaces must be designed to avoid information overload. While users can turn the various display features on and off individually, it is still easy in this demo for the display to become confusing. After listening to 3-D audio alerts for a while, some users found them fatiguing and distracting. Audio alerts must be used more sparingly. Once the driver finds the ambulance, he doesn't need the siren wailing anymore. This demo has seven aircraft overall, four of which are assumed to be equipped with ADS. Even with these few, the display can become cluttered in some visualization modes. A more realistic scenario might put 40-50 aircraft in the skies around the airport. Future versions of the demo need to be more sensitive to the potential for information overload.

\section{Free Flight Demonstration}

Based on the direction of other ATC work we are performing and the feedback from the domain experts, we are focusing on building visualization aids for Free 
Flight. In March 1996, the FAA decided to begin the process of shifting the current air traffic control system to a new policy of air traffic management called Free Flight $^{13}$. Free Flight shifts the emphasis from active to passive control, with a policy of intervention by exception $^{14}$. Under certain circumstances, aircraft will be allowed to select their own routes to their destinations, provided that they do not conflict with other aircraft. This freedom to set courses could result in significant time and financial savings for commercial airlines. When potential conflicts occur, Free Flight policies emphasize having aircraft attempt to resolve conflicts by themselves, with air traffic managers (who oversee dynamically-defined airspace sectors) intervening only when required.

What constitutes a conflict? To ensure safety, no aircraft should penetrate another aircraft's Protected Airspace Zone (PAZ): a cylindrical region around each aircraft with a 5 nautical mile radius and total height of 2000 feet (Figure 9). To avoid penetrating a PAZ, aircraft can change velocity, heading, or altitude. The Tactical Alert Zone (TAZ) is a region around an aircraft that defines the space where it is no longer possible to execute a maneuver to avoid penetrating the PAZ. For example, see Figure 10. Aircraft B is inside aircraft A's Velocity TAZ. That means even if both aircraft $A$ and B cooperate and optimally change their velocities, penetrating the PAZ is inevitable due to limitations on aircraft performance. However, aircraft $\mathrm{B}$ is still outside aircraft A's Heading TAZ, so it is possible to change headings and avoid penetrating the PAZ. In the worst case, if it is impossible to avoid penetrating the PAZ, then any available control (velocity, heading, altitude) must be used to maximize the miss distance.

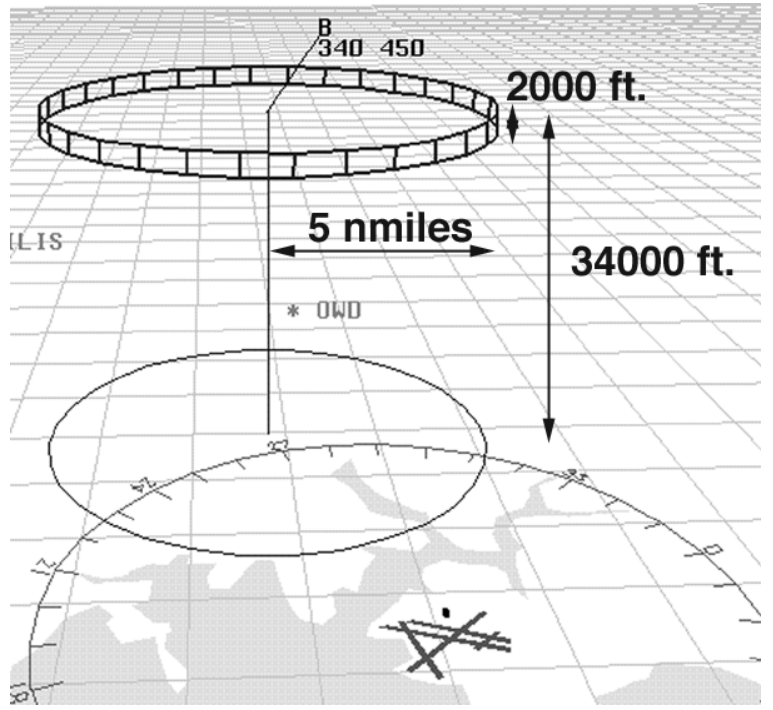

Figure 9: Protected Airspace Zone (PAZ).

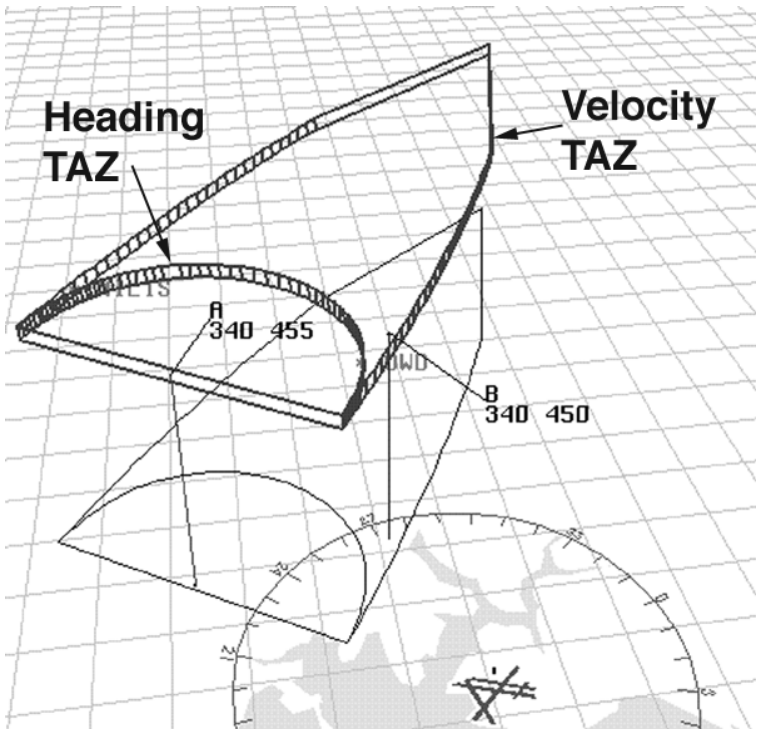

Figure 10: Tactical Alert Zones (TAZs).

The TAZ are complex, constantly changing shapes that are not easily explained or communicated without the use of 3-D graphics. Computing the shape of the TAZ requires knowledge of performance limits (maximum turn rate, maximum climb rate, etc.) and controllability and reachability constraints derived from the relative equations of motion. These computations are described in another paper ${ }^{10}$. The PAZ and TAZ are important safety regions that both pilots and air traffic managers must clearly understand to perform conflict detection and resolution in Free Flight. Both the PAZ and TAZ are centered on a particular aircraft and move with the aircraft. While the PAZ does not change shape with time, the TAZ will change depending on the relative positions, headings, speeds and conditions of the two aircraft. The TAZ cannot be easily generalized in terms of a heuristic that a pilot or air traffic manager can use in a non-visual mode. The best way to make use of the theoretical and algorithmic backgrounds behind computing the TAZ is to display the TAZ regions around the aircraft in a visual manner.

To illustrate TAZ regions for heading control manuevering, we have created a three-aircraft tactical conflict detection and resolution example. Three aircraft, labelled A, B, and C, are headed on courses that will cause them to penetrate each other's PAZ regions. These aircraft are too close to initiate speed control manuevers and are currently being told to stay within the same flight level by air traffic management. Figures 11-13 show what happens if the aircraft do not execute any heading changes. Figures 14-19 show how things change if the aircraft cooperate and adjust their 
headings to avoid penetrating each other's safety zones. In this case, the conflict resolution maneuver performed by aircraft $\mathrm{A}$ and $\mathrm{B}$ leads to a secondary conflict and subsequent resolution between $\mathrm{A}$ and $\mathrm{C}$, allowing for the safe passage of all three aircraft. In all these figures we see the TAZ regions around aircraft $B$ or $C$ with respect to possible penetration by aircraft $A$. Ghost planes extended 30 seconds into the future show the intended paths of each aircraft. By displaying what will happen with no course change, these visual displays provide advance warning of potential conflicts and allow the pilots and the air traffic managers to understand the control advice provided by the TAZ regions. This three-aircraft scenario is a particularly difficult multi-aircraft conflict that might occur under Free Flight. Investigating such scenarios provides a way to research multiple aircraft conflict detection and resolution algorithms and visualization techniques to aid situational awareness and the presentation of conflict resolution advisory information.

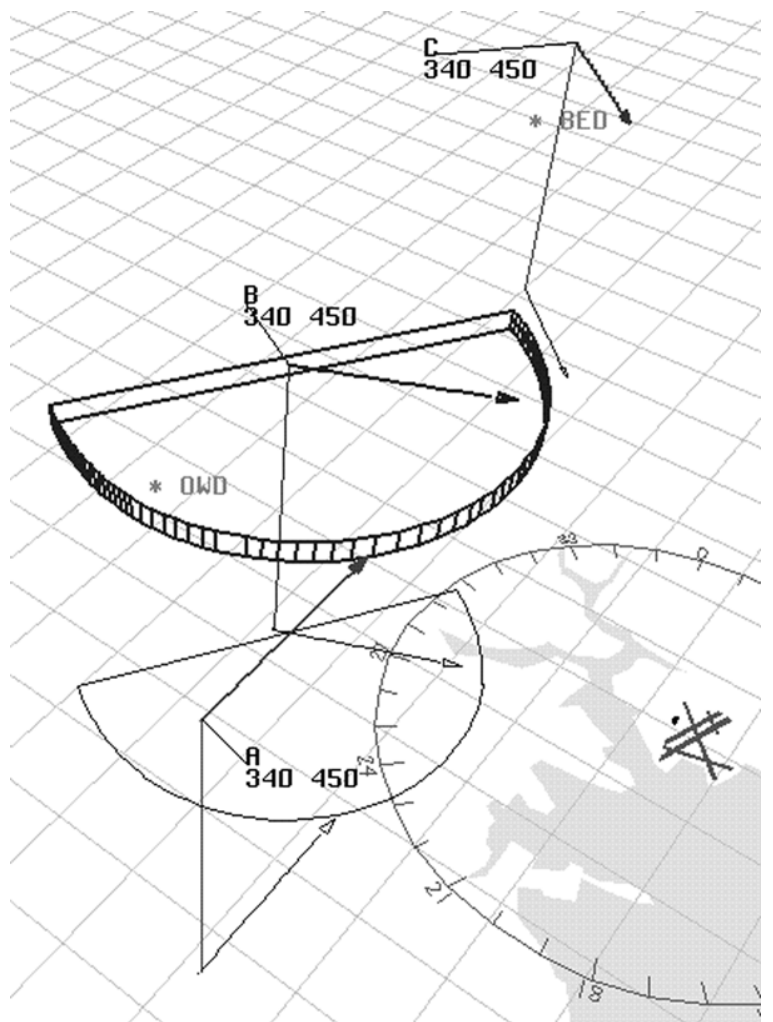

Figure 11: 3 aircraft scenario, no maneuvering. Time $=10$ seconds.

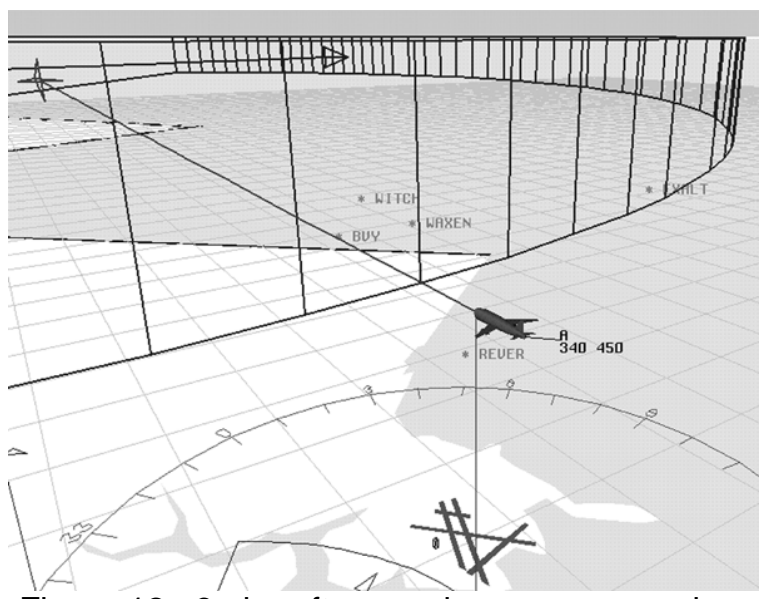

Figure 12: 3 aircraft scenario, no maneuvering. Time $=38$ seconds. Pilot A's view of impending contact with aircraft B's Heading TAZ.

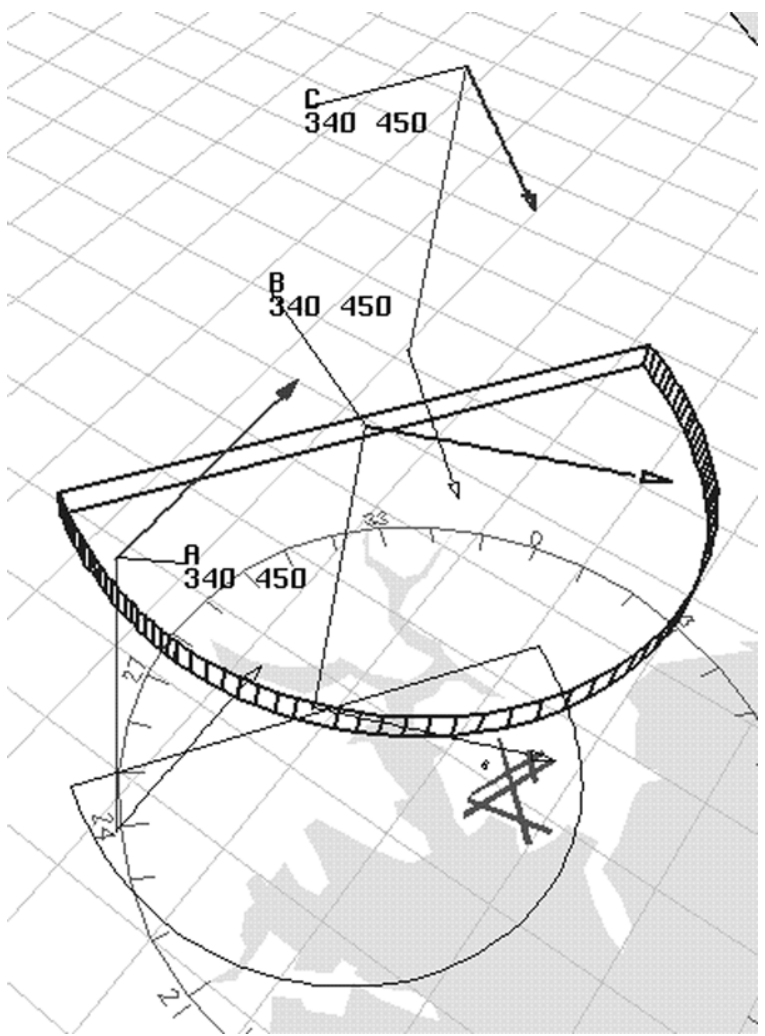

Figure 13: 3 aircraft scenario, no maneuvering. Time $=55$ seconds. Aircraft $A$ has penetrated aircraft B's Heading TAZ. 


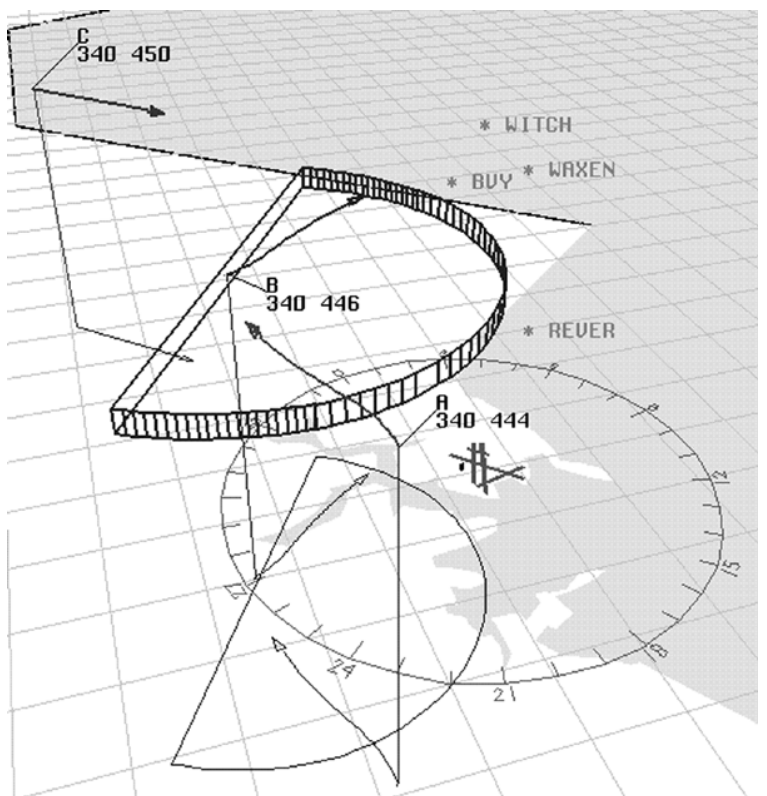

Figure 14: 3 aircraft scenario, with maneuvering. Time $=33$ seconds. Aircraft $A$ and $B$ both start turning to avoid penetration of B's TAZ.

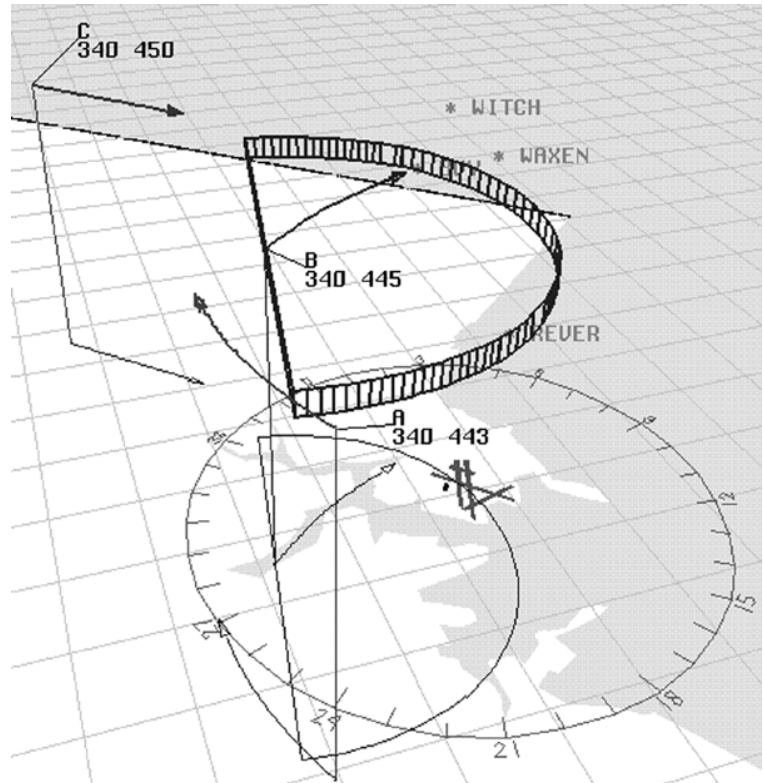

Figure 15: 3 aircraft scenario, with maneuvering. Time $=46$ seconds. Aircraft $A$ is almost finished clearing aircraft B's TAZ.

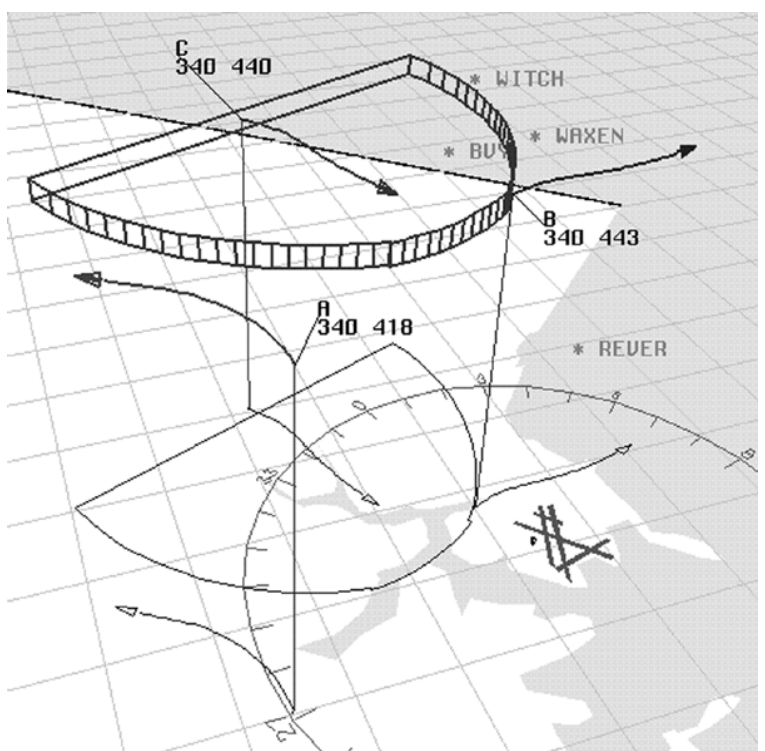

Figure 16: 3 aircraft scenario, with maneuvering. Time $=80$ seconds. Aircraft $A$ has cleared aircraft $B$ but is now approaching aircraft $C$. Aircraft $A$ and $\mathrm{C}$ begin cooperative turn to avoid penetration of C's TAZ.

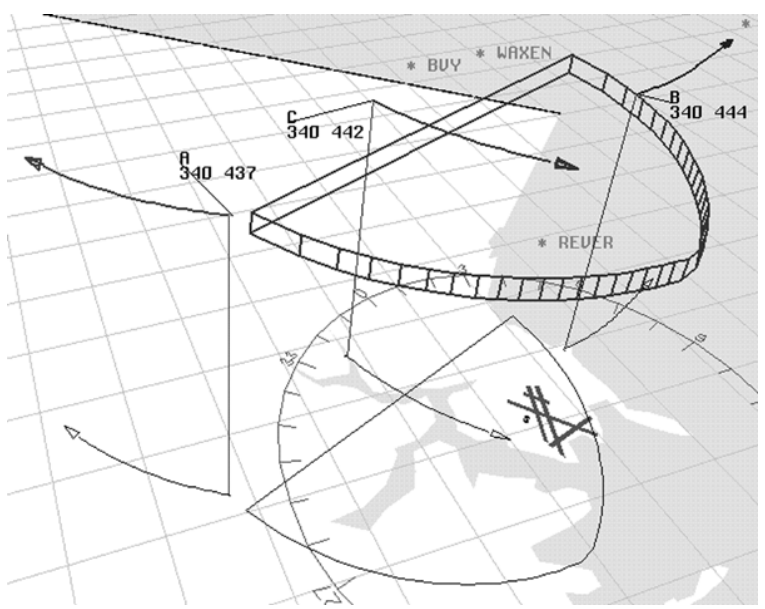

Figure 17: 3 aircraft scenario, with maneuvering. Time $=103$ seconds. Aircraft $A$ has just finished clearing aircraft C's TAZ.

Notes on Figures 11-18: All three aircraft stay at 34000 feet and are labelled A, B, and C on the diagrams. Altitude lines extend from the three aircraft positions to the ground. Shadows for the TAZ regions and the "ghost plane" lines are drawn on the ground. The lines extending from the aircraft positions and terminating in an arrow are the ghost plane lines. These visualizations are easier to see on the workstation monitor because the user can interactively change his viewpoint in real time and can see them as 3-D stereo images. 


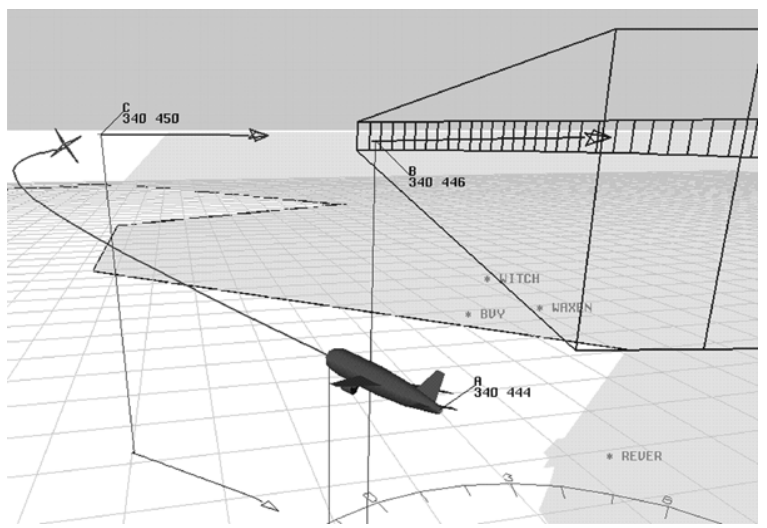

Figure 18: 3 aircraft scenario, with maneuvering. Time $=53$ seconds. Pilot A's view just after clearing aircraft B's TAZ.

\section{Future Work}

We have the following plans to continue developing visualization and interface techniques for aiding Free Flight:

- New hardware and software infrastructure: The existing demonstration is written in the $\mathrm{C}$ language using the GL graphics library. Consequently, adding new features requires changing low-level code, which is time consuming. We will switch to a higher-level software toolkit that is built for 3-D display and interaction, such as Sense 8's WorldToolKit or Division's dVISE library. This should result in faster changes and more rapid prototyping. We also anticipate moving to a more capable hardware platform, such as an SGI Infinite Reality, to avoid restricting our visualization ideas to the performance limitations of our SGI Indy. We will also build a version of the code that shows images on a wide field-of-view immersive stereo display. Hughes Research Labs has a 160 degree fieldof-view toroidal screen driven by three projectors. While not appropriate for pilots, the air traffic manager or AOC personnel of the future might stand in front of such a display and take advantage of the large visual display area provided. Such displays are sometimes referred to as CAVEs 6 .

- Improved interaction: The existing mouse-based interface may be satisfactory for a workstation-based demo, but as we move to large-screen displays this interface will no longer be appropriate. Selecting objects and changing modes should be allowed by issuing voice commands, rather than clicking objects on the display. Speech recognition has progressed to the point where small vocabulary speaker-independent recognizers, suitable for this task, are available on PCs. Head tracking will be required to correctly render the scene and allow user gaze to select objects. We will also track hand locations or handheld objects that the user manipulates to allow gesture-based control mechanisms.

- More realistic data: The current demonstration has only seven aircraft, flying routes that were not modelled according to existing flight paths or physical simulation. We will acquire more realistic databases of an airspace with flight routes from real commercial aircraft. We must populate the airspace with more aircraft, around 40 or 50 , to more realistically simulate the congestion that can exist around busy airports.

- Integration with conflict detection and resolution: Airspace congestion can lead to operator overload problems. To avoid this overload, filters will be used so that the user will only see the information that is critical. A pilot in Free Flight does not need to keep track of all neighboring aircraft, but rather only the ones that threaten to result in a conflict. Conflict detection and resolution algorithms can provide that information while improved visualization reduces operator overload.

- More visualization modes: More work needs to be done in exploring additional ways of representing the TAZ regions. What are the best ways to show the TAZ for heading, velocity, and altitude changes? The visualizations might be different for each type of zone.

\section{References}

[1] 40th Annual Air Traffic Control Association Conference. (Las Vegas, NV, 10-14 September 1995).

[2] Begault, D. R. Head-Up Auditory Displays for Traffic Collision Avoidance System Advisories: A Preliminary Investigation. Human Factors 35, 4 (December 1993), 707-717.

[3] Brown, Mark A. On the Evaluation of 3D Display Technologies for Air Traffic Control. Queen Mary \& Westfield College Dept. of Computer Science Technical Report No. 682 (16 August 1994).

[4] Brown, Mark A. 3D Display Technologies for Air Traffic Control: A Pilot Study. Queen Mary \& Westfield College Dept. of Computer Science Technical Report No. 690 (12 December 1994).

[5] Burnett, Meridyth Svensa and Woodrow Barfield. Perspective versus plan view air traffic control displays: Survey and empirical results. Proceedings of Human Factors Society 35th Annual Meeting (1991), 87-91. 
[6] Cruz-Neira, Carolina, Daniel J. Sandin, and Thomas A. DeFanti. Surround-Screen ProjectionBased Virtual Reality: The Design and Implementation of the CAVE. Proceedings of SIGGRAPH '93 (Anaheim, CA, 1-6 August 1993), 135-142.

[7] Daily, Michael J. and Jimmy A. Krozel. HumanCentered Interfaces for Air Traffic Control. Proceedings of the 40th Annual Air Traffic Control Assoication (ATCA) Conference (Las Vegas, NV, 10-14 September 1995), 215-219.

[8] Ellis, Stephen R. and Michael W. McGreevy. Perspective Traffic Display Format and Airline Pilot Traffic Avoidance. Human Factors 29, 2 (August 1987), 371-382.

[9] Haskell, I.D. and Christopher D. Wickens. Twoand Three-Dimensional Displays for Aviation: A Theoretical and Empirical Comparison. International Journal of Aviation Psychology 3, 2 (1993), 87-109.

[10] Krozel, Jimmy, T. Mueller and G. Hunter. Free Flight Conflict Detection and Resolution Analysis. To be published in Proceedings of AIAA Guidance, Navigation and Control conference (San Diego, CA, 29-31 July 1996).

[11] May, Patricia A., Margaret Campbell and Christopher D. Wickens. Perspective Displays for Air Traffic Control: Display of Terrain and Weather. Air Traffic Control Quarterly 3, 1 (1995), 1-17.

[12] Moller, H. and G. Sachs. Synthetic Vision for Enhancing Poor Visibility Flight Operations. IEEE AES Systems Magazine (March 1994), 2733.

[13] Nomani, Asra Q. FAA to Let Pilots Change Flight Paths. The Wall Street Journal (15 March 1996), A3.

[14] Nordwall, Bruce D. Free Flight: ATC Model for the Next 50 Years. Aviation Week \& Space Technology (31 July 1995), 38-39.

[15] Pruyn, Peter W. and Donald P. Greenberg. Exploring 3D Computer Graphics in Cockpit Avionics. IEEE Computer Graphics and Applications (May 1993), 28-35.

[16] Scardina, John A., Theodore R. Simpson, and Michael J. Ball. ATM: The only constant is change. Aerospace America (March 1996), 20-23 and 40.

[17] Smith, J.D., Stephen R. Ellis and Edward Lee. Avoidance Manuevers Selected While Viewing Cockpit Traffic Displays. NASA Ames Research Center technical report TM-84269 (October 1982), 34 pages.
[18] Ventrella, Jeffrey and Robert McNeil. MIT Media Laboratory, personal communication (1994).

[19] Wickens, C. D., S. Todd and K. Seidler. ThreeDimensional Displays: Perception, Implementation, and Applications. University of Illinois at Urbana-Champaign Aviation Research Laboratory technical report ARL-89-11 (1989).

[20] Zeltzer, David and Steven Drucker. A Virtual Environment System for Mission Planning. Proceedings of the IMAGE VI conference (Scottsdale, AZ, 14-17 July 1992), 125-134. 